\title{
The increase in copeptin levels in mild head trauma does not predict the severity and the outcome of brain damage
}

\author{
Luigi M Castello*,1,2, Livia Salmi ${ }^{1}$, Isabella Zanotti ${ }^{2}$, Clara A Gardino ${ }^{1}$, Marco Baldrighi ${ }^{1}$, \\ Fabio Settanni ${ }^{3}$ \& Gian C Avanzi $i^{1,2}$ \\ ${ }^{1}$ Department of Translational Medicine, Università del Piemonte Orientale UPO, via Solaroli, 17. 28100 - Novara, Italy \\ ${ }^{2}$ AOU Maggiore della Carità, Corso Mazzini, 18. 28100 - Novara, Italy \\ ${ }^{3}$ Division of Endocrinology, Diabetology \& Metabolism, Department of Medical Sciences, Università di Torino, Corso AM Dogliotti, \\ 14. 10126 - Torino, Italy \\ *Author for correspondence: Tel.: +390321 3733097; luigi.castello@med.uniupo.it
}

\begin{abstract}
Aim: To investigate the copeptin prognostic role in mild head trauma. Methods: We enrolled 105 adult patients who entered the emergency room because of recent mild head trauma; we evaluated: clinical picture, imaging and laboratory data (including copeptin). Results: Copeptin resulted higher in mild head trauma patients compared with controls: $29.89 \mathrm{pmol} / \mathrm{l}$ versus $7.05 \mathrm{pmol} / \mathrm{l}(\mathrm{p}=0.0008)$. Copeptin failed in identifying patients with or without brain lesions detected by CT scan, and patients with or without adverse events during the 30 days follow-up. Conclusion: We confirmed that mild head trauma patients have a significantly higher copeptin plasma levels compared with controls. Nevertheless, we did not observe a significant role for copeptin in traumatic brain injury patients regarding brain damage and outcome.
\end{abstract}

First draft submitted: 31 January 2018; Accepted for publication: 16 March 2018; Published online: 5 April 2018

Keywords: biomarker $\bullet$ copeptin $\bullet$ copeptin prognostic value $\bullet$ TBI $\bullet$ traumatic brain injury

Traumatic brain injury (TBI) is defined as brain dysfunction caused by mechanic injury and it is the leading cause of death in the young population; it has been estimated that in the USA about $2 \%$ of general population lives with disabilities caused by TBI [1], while in Europe the estimates are slightly lower, but still relevant [2].

Until now, Glasgow Coma Scale (GCS) is the only clinical score universally accepted to classify TBI. The age of the patient and initial severity of the injury, as detected by CT scan, are independent predictors of survival after TBI. However, these clinical factors are sometimes not accurate enough to predict outcomes after TBI in individual patients [3]. For these reasons several research groups investigated new biomarkers that could offer a more accurate prediction of patients' outcomes.

Copeptin is the C-terminal portion of preprovasopressin which is secreted by posterior pituitary gland in response to osmotic and hemodynamic variations; copeptin is secreted with equimolar ratio with arginine vasopressin (AVP) but, unlike the latter, it is characterized by a great stability and is therefore easily measurable in plasma [4].

Copeptin in the last decade has achieved some relevance as prognostic biomarker in different diseases such as acute coronary syndrome [5], heart failure [6] and acute exacerbation of chronic obstructive pulmonary disease [7]. In the last years, several studies investigated a potential role of copeptin also in different neurological damages; Zhu $\mathrm{XD}$ and colleagues, in 2011, showed that copeptin level in plasma was able to predict the outcome and the degree of cerebral vasospasm in subarachnoid hemorrhages $(\mathrm{SAHs})$ patients $(\mathrm{n}=300)[8]$.

Furthermore, in a study with 120 patients with acute cerebral hemorrhage, copeptin was found to be correlated with the severity and with 1 year mortality. Usefully, copeptin seems to enhance the NIH Stroke Score (NIHSS) prognostic power in these patients [9].

In 2017, through a systematic review and meta-analysis, Choi et al. [3] focused on copeptin prognostic role about the functional outcome and mortality in patients with TBI.

Despite all these studies, the role of copeptin as prognostic marker is still debated. Shortly after Choi publication, Veisani et al. [10] commented on Choi's review highlighting a low studies heterogeneity included in the meta-analysis.

Future Medicine 
The same authors underlined how many studies with negative results may have been conducted and not published at all.

With the aim to demonstrate the possible usefulness of Copeptin in mild TBI in emergency room (ER) we performed a prospective study; here we report our findings on plasmatic concentration levels of copeptin in mild TBI patients and its potential role as a prognostic factor.

\section{Methods}

\section{Study design \& patient selection}

We performed a longitudinal, prospective and observational study in the ER and in the observation unit of the emergency department of the 'Maggiore della Carità' University Hospital of Novara (Italy). The study was approved by the local ethical committee. We enrolled adult patients who entered the ER for mild TBI (GCS $\geq 13$ ) within 8 h prior to access, with or without minor trauma of the limbs. Exclusion criteria were previous TBI in the 3 months prior to the enrolment, clinically relevant thoracic or abdominal trauma or signs of hemorrhagic shock and history of stroke, meningitis and vasculitis with cerebral involvement in the previous 3 months.

All patients were asked to give a written informed consent for their participation in the study, which was conducted in strict adherence to the principles of the Declaration of Helsinki.

At the entrance in the ER, the GCS score and the main medical and clinical information were collected, with particular focus on the dynamics of the traumatic event, the daily therapy with antiplatelet and/or anticoagulant drugs, the clinical manifestations (syncope, headache, nausea, vomiting, dizziness, seizures, amnesia, diplopia or alterations of the visual field), the alterations of the neurological state (altered mental status, drowsiness, cognitivemotor slowing slurred speech, impaired walking, lack of motor coordination, paralysis and other abnormal findings on neurological examination).

Patients underwent a cerebral CT scan immediately after the ER admission or after an observation time of $6 \mathrm{~h}$, as indicated by good clinical practice. If clinically indicated, the CT scan could be repeated after a $24 \mathrm{~h}$ observation period.

A 30 days phone call follow-up was performed by an ER physician with a structured interview exploring the occurrence of adverse events (death or ER readmission) and the presence of symptoms of TBI. Patients were then considered to have a 'positive follow-up' if either an adverse event occurred or at least one symptom was reported.

As a control group, we dosed plasma copeptin in patients who entered the ER for nonacute conditions in the absence of recent traumatic event, respiratory failure, congestive heart failure or severe hypo and hyperosmolality. TBI patients and control group were matched for median age and sex. Since the exam was not clinically indicated, head CT scan was not performed in controls.

\section{Copeptin measurement}

A blood sample was collected and stored in ethylenediaminetetraacetic acid for the determination of plasmatic copeptin concentration at time of enrolment. Blood samples were centrifuged at room temperature for $10 \mathrm{~min}$ at 3000 r.p.m. within $1 \mathrm{~h}$ from the collection. Aliquots of the centrifuged blood samples were then stored at $-80^{\circ} \mathrm{C}$, and the copeptin assays were performed at the end of the study period at the Laboratory of Endocrinology of 'Città della Salute e della Scienza', University of Torino (Italy), by automated immunofluorescence assay using the BRAHMS Copeptin proAVP assay (Thermo Fisher Scientific, MA, USA).

\section{Statistical analysis}

Discrete variables have been described as ratio and frequency, while continuous variables are described as median with interquartile range. Comparisons between discrete variables were made by $\chi^{2}$ tests, while continuous tests with Mann-Whitney U test and Kruskal-Wallis test. The statistical significance level was set at a p-value $<0.05$.

\section{Results}

We enrolled 105 cases and 22 controls from July 2014 to March 2015. The main characteristics of the study population are summarized in Box 1. Particularly, most of the patients had a GCS score at presentation of 15, since only one patient had a score of 14 due to eye-opening at the call. Twenty-one patients had a positive CT scan at ER evaluation. The computed tomography (CT) findings were distributed as follow: six petechial hemorrhages, four SAH, seven subdural hematoma and four epidural hematoma (no significant differences in copeptin levels were found among these four groups using Kruskal-Wallis test). Lesions size could not be analyzed since the extent 
Box 1. Main characteristics of traumatic brain injury patients.

\section{Age}

- Median: 77 years old

- IQR: 59-83 years old

Sex

- Female: $66 / 105(62.9 \%)$

- Male: 39/105 (37.1\%)

Antiplatelet and/or anticoagulant drugs

- None: 58/105 (55.2\%)

- One or more antiplatelet agent: $30 / 105$ (28.7\%)

- Oral anticoagulant drug: 16/105 (15.2\%)

- Both anticoagulant and antiplatelet drug: 1/105 (0.9\%)

Dynamic of the event

- Low dynamic: 79/105 (75.2\%)

- High dynamic: $26 / 105$ (24.8\%)

Symptoms at presentation ${ }^{\dagger}$

- None: 61/105 (58.1\%)

- At least one symptom: 44/105 (41.9\%)

Neurological signs at presentation ${ }^{\dagger}$

- None: $80 / 105$ (76.2\%)

- At least one neurological sign: 25/105 (23.8\%)

GCS score at presentation

- 15/15: 104/105 (99.1\%)

- $14 / 15$ ( $E=3$, 'opens his eyes at the call'): $1 / 105(0.9 \%)$

Blood pressure

- Median: $140 / 80 \mathrm{mmHg}$

- IQR: $128 / 70-160 / 90 \mathrm{mmHg}$

Brain CT scan at time zero

- No evidence of bleeding lesions: $84 / 105$ (80.0\%)

- Minor ${ }^{\ddagger}$ bleeding lesions: 21/105 (20.0\%)

- Petechial parenchymal haemorrhages: $6 / 21$ (28.7\%)

- Subarachnoid haemorrhage: 4/21 (19.0\%)

- Subdural haematoma: $7 / 21(33.3 \%)$

- Epidural haematoma: 4/21 (19.0\%)

30 days follow-up

- Responders: 84/105 (80.0\%)

- Negative follow-up (no clinical events TBI-related): 50/84 (59.4\%)

- Positive follow-up: $34 / 84(40.6 \%)$

- TBI-related symptoms: $33 / 34(97.1 \%)$

- ER readmission: 22/34 (64.7\%)

- Death: $1 / 34$ (2.9\%)

- Lost to follow-up: $21 / 105(20.0 \%)$

${ }^{\dagger}$ The absolute frequencies of each investigated sign and symptom are reported in Figure 1.

¥All the observed intracranial lesions were Grade 2 according to the Marshall CT Classification of TBI (i.e., cisterns present, no midline shift $>5 \mathrm{~mm}$, no lesions $>25 \mathrm{~cm}^{3}$ ).

CT: Computed tomography; ER: Emergency room; GCS: Glasgow Coma Scale; IQR: Interquartile range; TBI: Traumatic brain injury.

of petechial hemorrhages and SAH is not easily measurable. However, regardless of the nature of the different lesions, all the CT findings were classified as Grade 2 according to the Marshall CT Classification of TBI (which is considered the best radiological prognostic predictor in TBI patients): assuming this classification to be a surrogate of size, all the observed lesions may be considered of the same size. Parts of these are included in Box 1.

None of the TBI patients sustained other clinically relevant traumatic injuries (e.g., bone fractures, extended limb wounds, etc.).

Figure 1 reports the absolute frequencies of each symptom and sign in TBI patients, while Figure 2 reports the frequencies of each TBI-related symptom investigated at follow-up. 


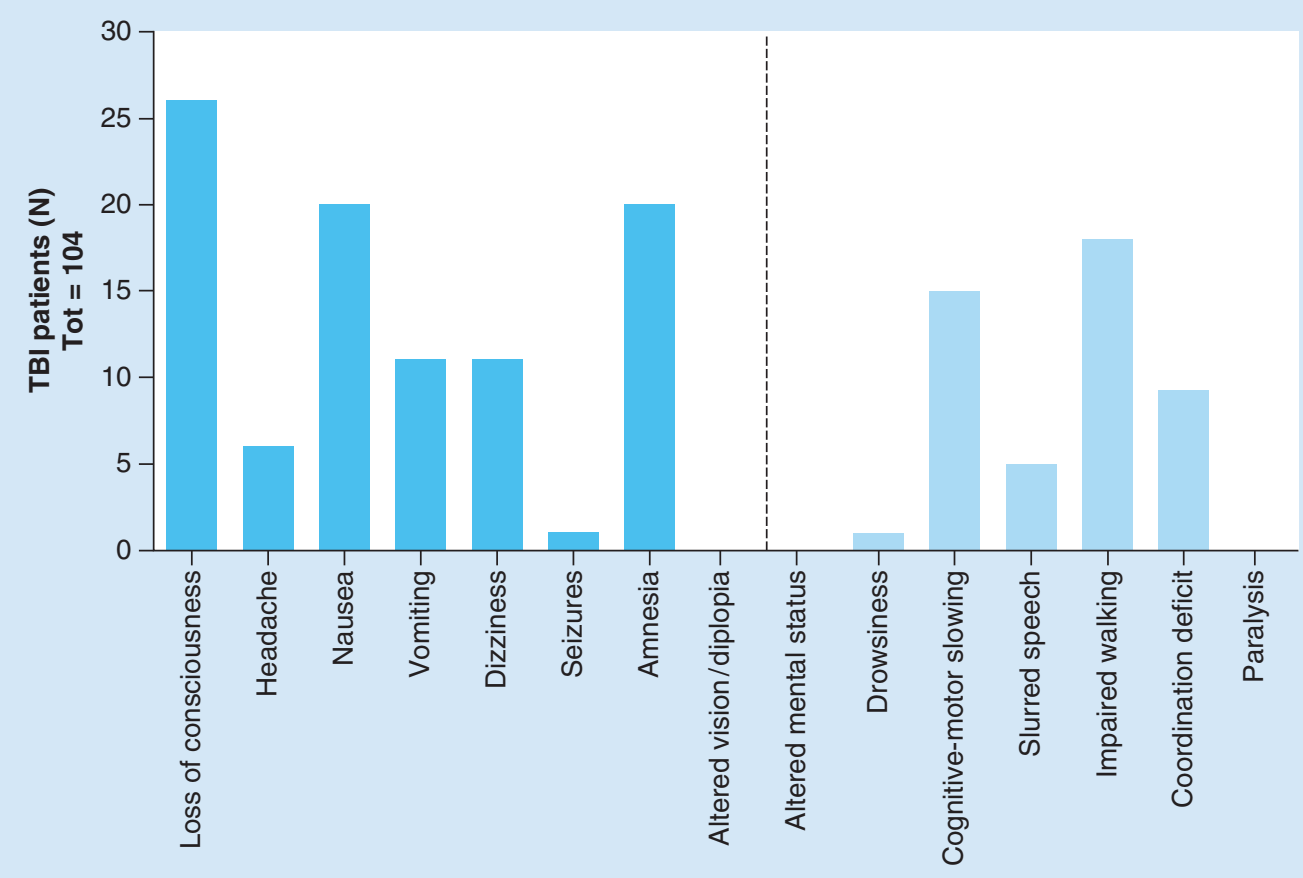

Figure 1. Absolute frequencies of symptoms and signs at presentation. Grey bars on the left of the vertical dashed line indicate symptoms while white bars on the right indicate signs. Part of the 'impaired walking' was related to cognitive-motor slowing, part to coordination deficits; no significant orthopedic injury, as well as spinal cord contusion occurred. No patient referred altered vision /diplopia. Altered mental status and paralysis were not detected in our patients.

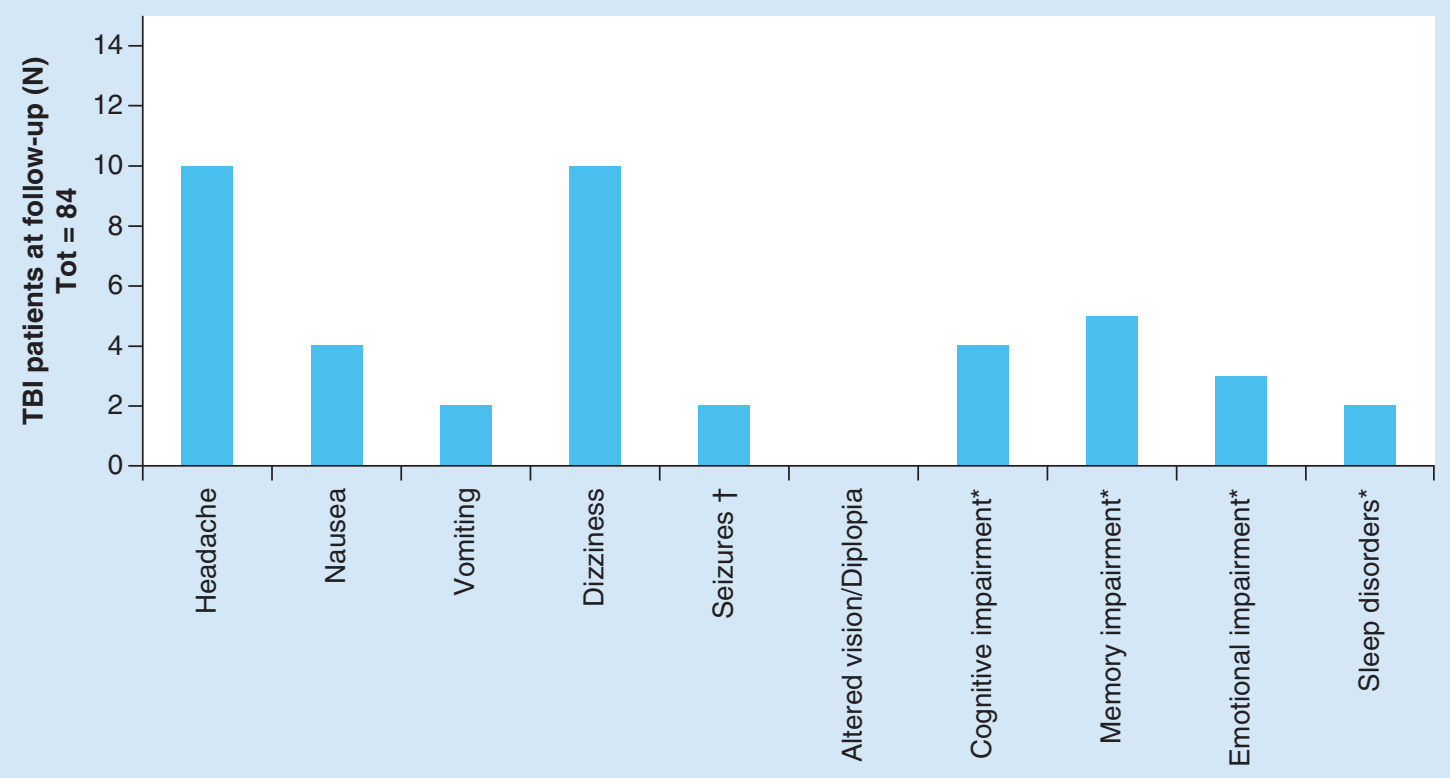

Figure 2. Absolute frequencies of traumatic brain injury-related symptoms reported at the follow-up interview. Altered vision/diplopia were referred by no patient during the follow-up interview.

* Only new-onset impairment or a worsening of a pre-existing impairment has been considered.

$\dagger$ One out of the two patients reporting seizures at follow-up already had a history of seizures before head trauma occurred. 


\begin{tabular}{|c|c|c|c|}
\hline \multirow[t]{2}{*}{ Variable } & \multicolumn{2}{|c|}{ Plasma copeptin (pmol/l) } & \multirow[t]{2}{*}{ p-value } \\
\hline & Median & IQR (25-75) & \\
\hline Age $>77(n=52 / 105)$ & 33.12 & $10.99-107.4$ & \\
\hline Male $(n=39 / 105)$ & 38.80 & $8.83-66.12$ & 0.7882 \\
\hline Female $(n=66 / 105)$ & 21.12 & $9.41-69.68$ & \\
\hline $\begin{array}{l}\text { At least one symptom or sign at ER visit } \\
(n=36 / 105)\end{array}$ & 20.93 & $11.12-68.01$ & \\
\hline Negative CT scan ( $n=84 / 105)$ & 21.89 & $9.44-65.99$ & 0.5970 \\
\hline Positive CT scan ( $n=21 / 105)$ & 34.52 & $8.16-107.4$ & \\
\hline Positive follow-up $(n=50 / 84)$ & 27.04 & $9.47-65.40$ & 0.8482 \\
\hline
\end{tabular}

The median value of plasma copeptin in mild TBI patients at enrolment time was $29.89 \mathrm{pmol} / \mathrm{l}(9.29-67.71)$ in comparison to the plasma copeptin values in controls that were found to be $7.05 \mathrm{pmol} / \mathrm{l}(3.4-18.8),(\mathrm{p}=0.0008)$ (Figure 3A).

The analysis of copeptin concentrations was conducted dividing patients into different groups based on age (cut-off value set at 77 years, the median age of our population), sex, presence of at least one symptom related to TBI or one alteration at the neurological examination, the presence (positive) or absence (negative) of abnormal findings on head CT scan and the outcome during the 30 days follow-up. The statistical analysis on demographic and clinical parameters showed no significant differences between these groups, as reported in Table 1. In particular, copeptin concentrations in negative CT patients and in positive CT patients were $21.89 \mathrm{pmol} / \mathrm{l}$ (9.44-65.99) and 34.52 (8.16-107.4), respectively, with a p-value of 0.5970 at Mann-Whitney $U$ test (Figure 3B). Dividing patients on the basis of follow-up outcome, copeptin levels were $27.04 \mathrm{pmol} / \mathrm{l}(9.47-65.40)$ and $22.79 \mathrm{pmol} / \mathrm{l}(8.17-75.98)$ for positive and negative follow-up patients, respectively, with a not significant p-value.

The study ended with the structured interview at 30 days, and the only deceased patient was a 93 year-old woman with multiple cardiovascular comorbidities, whose death cannot be directly attributed to TBI; her copeptin value at $\mathrm{ER}$ admission was $5.34 \mathrm{pmol} / \mathrm{l}$.

A collateral analysis focused on potential prognostic predictors other than copeptin in our population showed that a positive 30 days follow-up could be predicted by the presence of at least one neurological sign at presentation (odds ratio [OR]: $13.96 ; 95 \% \mathrm{CI}: 3.62-53.58 ; \mathrm{p}=0.0001$ ), by the presence of at least one symptom at presentation (OR: 2.53 ; 95\% CI: $1.05-6.37 ; \mathrm{p}=0.048$ ) and by a positive CT imaging (OR: 5.57; 95\% CI: 1.7568-17.6693; $\mathrm{p}=0.003)$.

\section{Discussion}

Many experimental studies, based on animal models of brain injury, demonstrated the implication of AVP in the development of cerebral edema, intracranial hypertension and electrolyte derangements. Molnàr et al. demonstrated that the administration of an AVP antagonist was effective in reducing cerebral edema in a mouse model of cerebral ischemia [11]. Two different experimental mouse models of head injury with cortical-controlled impacts showed that intracerebral administration of the V1a AVP receptor antagonist reduces the cerebral content of water and the cerebral contusion extension [12,13].

These findings led to the hypothesis that AVP secretion could be used as a marker to assess the severity of the brain injury. Since AVP is highly unstable, many authors preferred to study copeptin instead of AVP. A strong correlation between copeptin plasma concentration and unfavorable outcome was found in atraumatic parenchymal cerebral hemorrhage $[8,14,15]$ and in ischemic stroke $[15,16]$. Moreover, in patients with severe head injury, plasmatic copeptin concentration has been shown to be significantly correlated with the severity of trauma, the initial GCS and the mortality. These results confirmed that copeptin is a predictor of unfavorable outcome in patients with severe head trauma and could be used as a prognostic biomarker $[17,18]$. Similar results have been obtained in a study involving a pediatric population [19]. Although these findings support the role of copeptin in the prognostic 
(A)

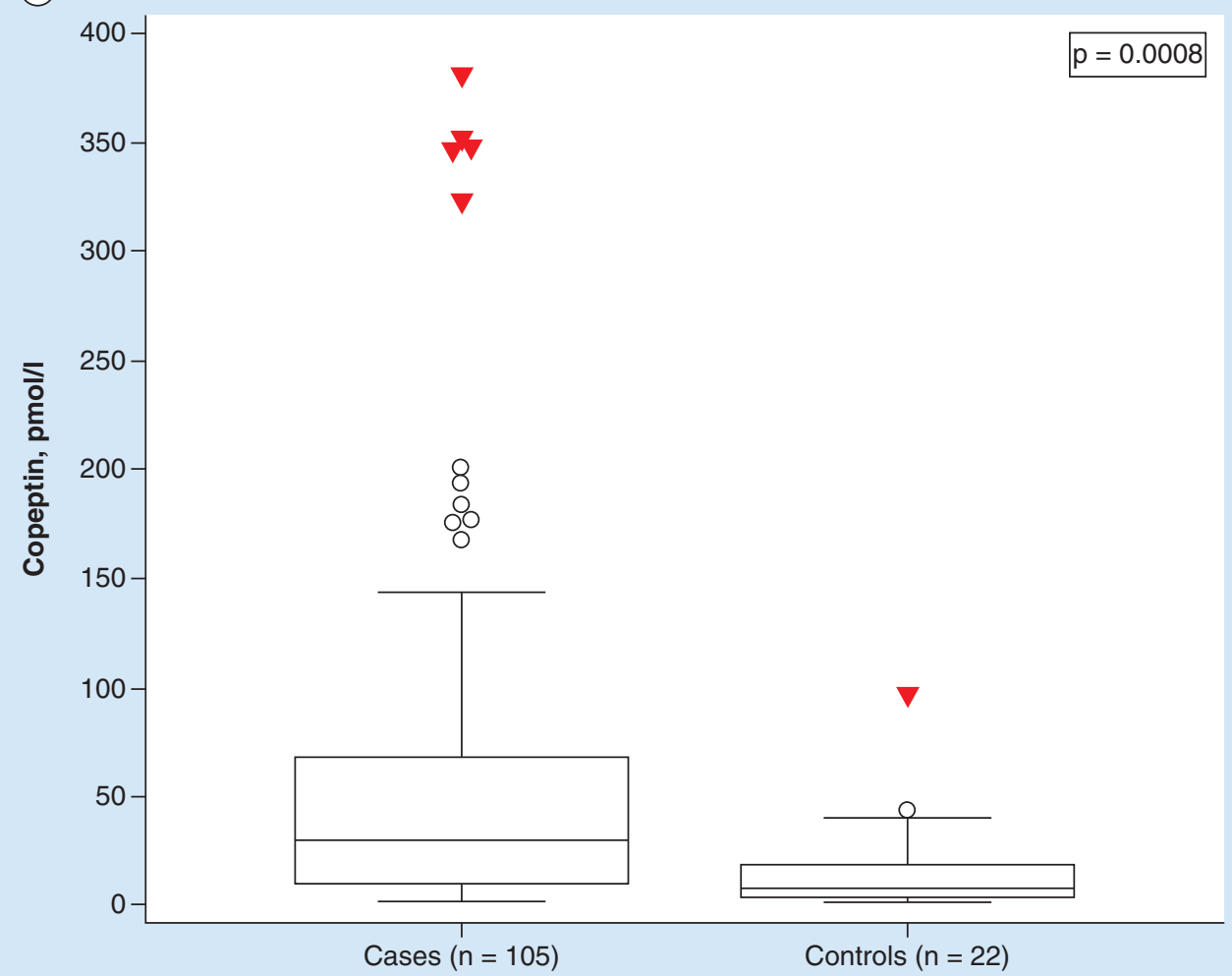

(B)

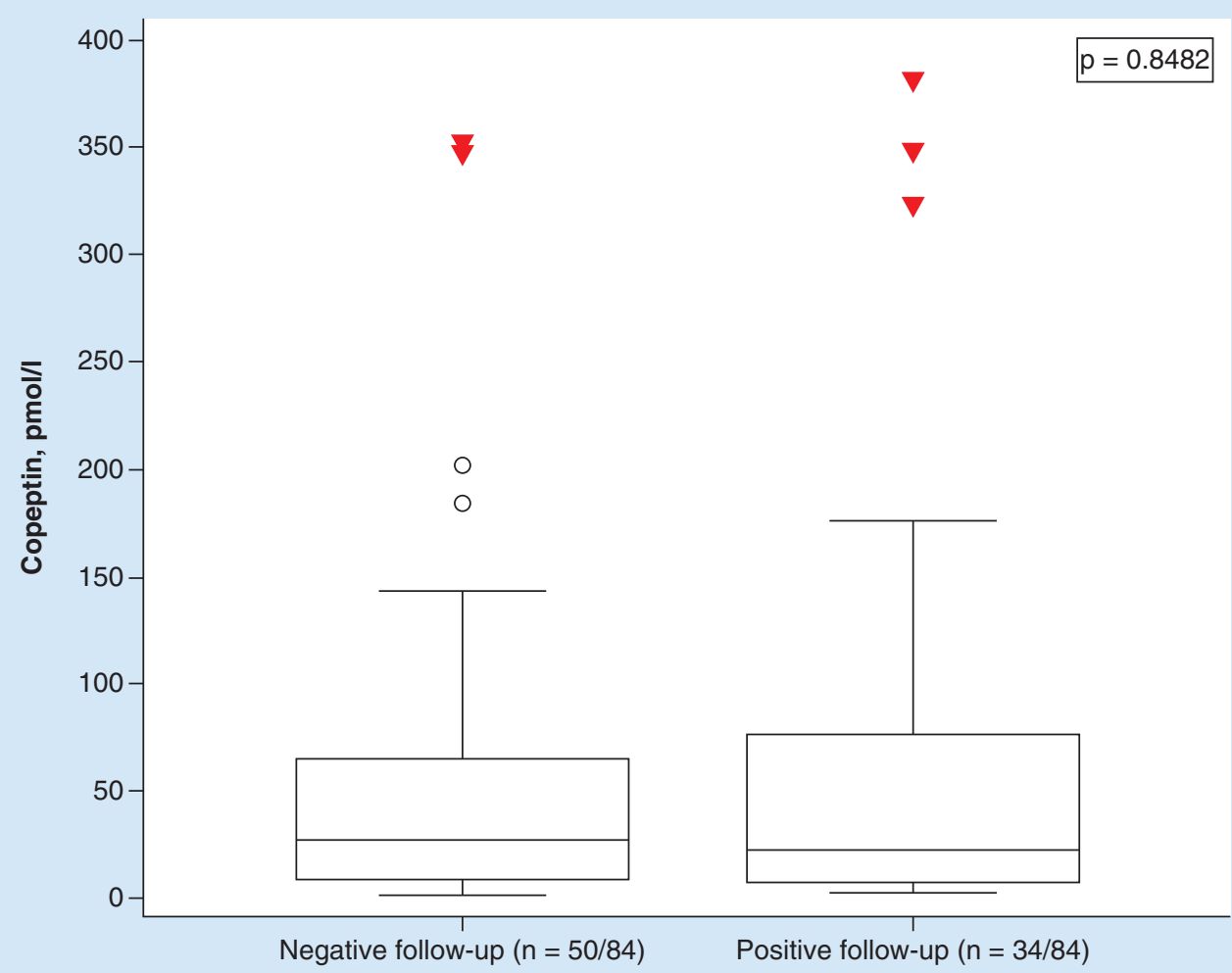

Figure 3. Analysis of plasmatic copeptin levels. (A) Comparison of plasmatic copeptin levels between traumatic brain injury (TBI) patients and controls. Copeptin levels were found to be significantly higher in TBI patients compared with controls $(p=0.0008)$. The graph also highlights the high variability observed in copeptin levels in TBI patients (circles indicate outside values and triangles indicate far out values). (B) Comparison of plasmatic copeptin levels between TBI patients with a positive follow-up and TBI patients with a negative follow-up. No significant difference was found in copeptin levels at emergency room admission between patients in the two groups. 
stratification of patients with severe TBI, poor data are available about the same role of copeptin in patients with mild and moderate TBI.

Herein, we report a study conducted on a cohort of 105 patients with mild TBI; plasma copeptin concentration was determined at ER admission and, since copeptin levels vary with sex [10], patients were matched with a control group by median age and sex to avoid confounders.

In this cohort, we observed a statistically significant higher copeptin concentration in plasma of mild TBI patients compared with controls. Although neuropsychological stress is an important stimulus for AVP secretion, we are confident that this phenomenon has not altered our results; patients included in the control group, in fact, were enrolled in the ER as well as head trauma patients and they experienced a certain degree of anxiety and stress related to their different clinical conditions. The above reported result is in line with the recently published meta-analysis, by Choi and colleagues, reporting elevated copeptin concentrations in severe TBI patients [3]. However, in our study, we did not identify any statistically significant difference in copeptin distribution among different groups of severity. Moreover, the prognostic performance of copeptin, evaluated by the presence of adverse events or death during a 30 days follow-up, turned out to be poor. In our population, the only strong predictors of the persistence of TBI-related symptoms with or without ER readmissions were the presence of at least one sign at presentation (OR: 13.96), the presence of at least one symptom (OR: 2.53) and a positive head CT scan for brain injury (OR: 5.57).

The poor prognostic value of copeptin in our study could be explained by the enrolment setting: in fact, our protocol scheduled a very early evaluation of patients, a few minutes after the ER admittance; in this setting many confounders may be present and may affect AVP secretion and, consequently, copeptin plasma concentration. Pain, nausea, vomiting, fear and anxiety are powerful stimuli for AVP secretion and we cannot exclude that these elements, often present in our cohort, could have affected the study results. Nevertheless, since this is a real-life protocol, we think that our data are strong enough to discourage copeptin use as a biomarker for the early risk stratification of mild head trauma patients evaluated in the ER. More studies are required to establish whether a different timing for copeptin assessment (e.g., at discharge, after observation) could improve its prognostic performance in mild TBI patients.

Limits: in the control group we did not include any patients referred to the ER because of trauma with no head involvement; probably the presence of such patients would have been useful to improve the interpretability of our results.

\section{Conclusion}

Although copeptin levels were found to be higher in patients with mild head trauma compared with controls, a single copeptin determination at ER admission showed no utility in the early stratification of mild head trauma patients.

\section{Future perspective}

Since our results did not confirm the prognostic role of copeptin in mild head trauma, we think that there is a lack of evidence supporting the future use of copeptin in this setting. We deem that further large observational studies should be performed in the future, in order to strenghten the level of evidence supporting the use of plasmatic copeptin levels for the prognostic stratification of patients with moderate to severe head trauma.

\section{Acknowledgements}

The present study was founded by the Translational Medicine Department, University of Piemonte Orientale (Novara, Italy). 
Summary points

- Plasmatic copeptin concentration has been previously correlated to the outcome in severe head trauma.

- The aim of this study was to evaluate the prognostic power of a single copeptin determination in mild head trauma.

- A total of 105 patients were enrolled.

- 21 patients had a positive CT scan.

- Copeptin levels were significantly higher in traumatic brain injury patients compared with controls.

- Copeptin levels were not significantly different between patients with a positive CT scan and with a negative CT scan.

- Copeptin levels were not significantly different between patients with a positive follow-up and with a negative follow-up.

- A positive CT scan or the presence of at least one symptom at presentation or the presence of at least one sign at presentation was strong predictor of a positive follow-up.

Ethical conduct of research

The authors state that they have obtained appropriate institutional review board approval or have followed the principles outlined in the Declaration of Helsinki for all human or animal experimental investigations. In addition, for investigations involving human subjects, informed consent has been obtained from the participants involved.

Open access

This work is licensed under the Attribution-NonCommercial-NoDerivatives 4.0 Unported License. To view a copy of this license, visit http://creativecommons.org/licenses/by-nc-nd/4.0/

\section{References}

Papers of special note have been highlighted as: $\bullet$ of interest; $\bullet \bullet$ of considerable interest

1. Rutland-Brown W, Langlois JA, Thomas KE et al. Incidence of traumatic brain injury in the United States. J. Head Trauma Rehabil. 21(6), 544-548 (2003).

2. Tagliaferri F, Compagnone C, Korsic M et al. A systematic review of brain injury epidemiology in Europe. Acta Neurochir. (Wien.) 148(3), 255-268 (2006).

3. Choi K-S, Cho Y, Jang B et al. Prognostic role of copeptin after traumatic brain injury: a systematic review and meta-analysis of observational studies. Am. J. Emerg. Med. 35(10), 1444-1450 (2017).

-. A systematic review including six studies and 552 patients that demonstrated the early copeptin measurement correlates with outcome in traumatic brain injury patients.

4. Morgenthaler NG, Struck J, Jochberger S et al. Copeptin: clinical use of a new biomarker. Trends Endocrinol. Metab. 19(2), 43-49 (2008).

- A thorough review summarizing the role of copeptin in different conditions.

5. Maisel A, Mueller C, Neath S-X et al. Copeptin helps in the early detection of patients with acute myocardial infarction: primary results of the CHOPIN trial (copeptin helps in the early detection Of patients with acute myocardial infarction). J. Am. Coll. Cardiol. 62(2), $150-160$ (2013).

6. Vetrone F, Santarelli S, Russo V et al. Copeptin decrease from admission to discharge has favorable prognostic value for 90-day events in patients admitted with dyspnea. Clin. Chem. Lab. Med. 52(10), 1457-1464 (2014).

7. Winther JA, Brynildsen J, Høiseth AD et al. Prognostic and diagnostic significance of copeptin in acute exacerbation of chronic obstructive pulmonary disease and acute heart failure: data from the ACE 2 study. Respir. Res. 18(1), 184 (2017).

8. Zhu X-D, Chen J-S, Zhou F et al. Detection of copeptin in peripheral blood of patients with aneurysmal subarachnoid hemorrhage. Crit. Care. 15(6), R288 (2011).

9. Zhang A, Li J, Li X et al. The prognostic value of copeptin for acute intracerebral hemorrhage patients. Exp. Ther. Med. 5(2), 467-470 (2013).

10. Veisani Y, Nematollahi S, Khazaei S. Comments on prognostic role of copeptin after traumatic brain injury: a systematic review and meta-analysis of observational studies. Am. J. Emerg. Med. doi:10.1016/j.ajem.2017.11.026 (2017).

11. Molnár AH, Varga C, Berkó A et al. Inhibitory effect of vasopressin receptor antagonist OPC-31260 on experimental brain oedema induced by global cerebral ischaemia. Acta Neurochir. (Wien.) 150(3), 265-271 (2008).

12. Trabold R, Krieg S, Schöller K et al. Role of vasopressin V(1a) and V2 receptors for the development of secondary brain damage after traumatic brain injury in mice. J. Neurotrauma 25(12), 1459-1465 (2008). 
13. Krieg SM, Sonanini S, Plesnila N et al. Effect of small molecule vasopressin V1a and V2 receptor antagonists on brain edema formation and secondary brain damage following traumatic brain injury in mice. J. Neurotrauma 32(4), 221-227 (2015).

14. Zhang X, Lu X-M, Huang L-F et al. Copeptin is associated with one-year mortality and functional outcome in patients with acute spontaneous basal ganglia hemorrhage. Peptides 33(2), 336-341 (2012).

15. De Marchis GM, Katan M, Weck A et al. Copeptin adds prognostic information after ischemic stroke: results from the CoRisk study. Neurology 80(14), 1278-1286 (2013).

16. Zhang J-L, Yin C-H, Zhang Y et al. Plasma copeptin and long-term outcomes in acute ischemic stroke. Acta Neurol. Scand. 128(6), 372-380 (2013).

17. Yu G-F, Huang Q, Dai W-M et al. Prognostic value of copeptin: one-year outcome in patients with traumatic brain injury. Peptides 33(1), 164-169 (2012).

- An observational study demonstrating that copeptin correlates to 1-year mortality and functional outcome in severe head trauma.

18. Dong X-Q, Huang M, Yu W-H et al. Change in plasma copeptin level after acute spontaneous basal ganglia hemorrhage. Peptides 32(2), 253-257 (2011).

19. Lin C, Wang N, Shen Z-P et al. Plasma copeptin concentration and outcome after pediatric traumatic brain injury. Peptides 42, 43-47 (2013). 
